\title{
Tratamiento de la fibrosis pulmonar idiopática
}

\section{Treatment of idiopathic pulmonary fibrosis}

Carlos Eduardo Matiz, MD., FCCP.

Desde el 2000 se vienen intentando nuevas terapias para la fibrosis pulmonar idiopática (FPI). El conocimiento de la fisiopatología ha permitido el avance en el diagnóstico, fenotipos, curso de la enfermedad, biomarcadores y tratamiento de la fibrosis pulmonar idiopática, permitiéndonos tener una esperanza con los últimos resultados de los estudios INPULSIS y ASCEND del 2014. Esto ha permitido el diseño de nuevos protocolos fase II y III, con objetivos primarios y secundarios específicos similares, con objetivos subrogados como son la capacidad vital forzada (CVF), la mortalidad, la difusión, las exacerbaciones, y la caminata de 6 minutos, en tiempos de estudio de 52 semanas promedio, que permitieron sacar conclusiones con nivel de evidencia científica y también descartar medicamentos que no producían mejoría en la sobrevida, mortalidad y la CVF $(1,17)$.

Existen parámetros que nos demuestran la severidad y el peor pronóstico fisiológico de la enfermedad, que se correlacionan con la morbimortalidad de la misma cuando se diagnostica la fibrosis pulmonar idiopática. La guía ALAT del 2014 los describe como estáticos y dinámicos. Estáticos como el grado de disnea por mMRC de 3 o 4, la difusión de monóxido de carbono (DLCO) de menos del $40 \%$, la desaturación en la caminata de 6 minutos de menos del $88 \%$, la extensión de la fibrosis en la TACAR de más de 2 a 3 partes de panalización, la medición de la hipertensión pulmonar, parámetros gasimétricos de insuficiencia respiratoria y enfisema combinado con fibrosis. Los dinámicos son aumento del grado de disnea, caída de más del $10 \%$ de la CVF, caída de más del $15 \%$ de la DLCO en 6 meses y progresión de los síntomas (2).

Las causas de la fibrosis pulmonar idiopática todavía están por ser identificadas. Existen factores que contribuyen a su desarrollo como son causas virales, reflujo gastroesofágico, cigarrillo o exposición ambiental a partículas inhaladas, que afectan a una minoría de la población que por factores genéticos o de envejecimiento desarrollan la enfermedad. Estos factores pueden ser múltiples como
Médico internista, Neumólogo. Profesor asociado Medicina, Universidad El Bosque. Coordinador Comité enfermedad pulmonar intersticial difusa. Asociación colombiana de Neumología y Cirugía de Tórax. Director CEEP.

Correspondencia: Carlos Eduardo Matiz, correo electrónico: carlosmatiz@ hotmail.com

Recibido: 30/08/14. Aceptado: 10/09/14. 
inestabilidad genómica, mutación en los telómeros (TERT y TERC) y acortamiento de los mismos, muy similar al desarrollo del cáncer, mutación en el surfactante en la proteína $\mathrm{C}$ y A2, disbalance entre la fibrosis y las sustancias y células antifibróticas con una cicatrización inadecuada debida a una injuria persistente que va a producir coagulación extravascular, activación inmune, reclutamiento de fibroblastos, proliferación, activación de la síntesis de la matriz extracelular, produciendo fibrosis pulmonar y perdida de la función no solo a nivel intersticial sino a nivel alveolar (3).

Todos los tratamientos han sido dirigidos, debido a la heterogeneidad de la enfermedad, a múltiples y diversos objetivos para detener la progresión de la fibrosis pulmonar idiopática como son el mecanismo antioxidante, el fenómeno vascular y coagulante, las antiendotelinas, el fenómeno inmune, el reclutamiento de los fibroblastos, la activación y diferenciación de fibroblasto en miofibroblasto, la acumulación de la matriz y expresión de las enzimas oxidativas $(4,5)$.

La combinación de la $\mathrm{N}$-acetilcisteína, azatioprina y prednisona fue considerada el tratamiento de elección por más de diez años para la fibrosis pulmonar idiopática con base en el estudio IFIGENIA, debido a su carácter inmune y antioxidante que se tenía en ese momento de la enfermedad. La N-acetilcisteína y su carácter de antioxidante como un precursor del glutatión, estimulando su síntesis y restableciendo los niveles, mostró en este estudio una reducción en la disminución de la CVF y la DLCO, sin efecto en la sobrevida, con muchos interrogantes con respecto a la dosis, la alta descontinuación y la falta real de placebo en el grupo control (6), por lo cual dio para que se realizara los estudios PANTHER $1 \mathrm{y}$ 2. El PANTHER 1, un estudio programado para 60 semanas con objetivo principal de evaluar el cambio en la CVF con 236 pacientes con un aleatorización $1: 1,50 \%$ de los pacientes en la combinación (prednisona, azatioprina, $\mathrm{N}$-acetilcisteína) y $50 \%$ en placebo. Los resultados mostraron un aumento en la mortalidad de la combinación de 8 pacientes frente a $1(\mathrm{p}=0,001)$, no hubo cambio en la progresión de la enfermedad ni en la CVF y se evidenció aumento en la hospitalización de 23 pacientes en la combinación frente a 7 en placebo ( $\mathrm{p}=0,001)$, por lo que decidieron suspender el estudio a las 32 semanas (7).

El PANTHER 2 fue la continuación con 264 pacientes con la aleatorización $1: 1, \mathrm{~N}$-acetilcisteína frente a placebo por 60 semanas, con objetivo principal de evaluar la variación en la CVF y objetivos secundarios la variación en la difusión, exacerbaciones, caminata en 6 minutos y cuestionarios de calidad de vida. Los resultados del estudio no encontraron diferencia significativa en el cambio de la CVF entre la $\mathrm{N}$-acetilcisteína y placebo $(-0,18$ litros y $-0,19$ litros) respectivamente $(\mathrm{p}=0,77)$, no diferencia en las tasas de mortalidad ( 4,9 vs. $2,5 \%, p=0,30)$ o de exacerbación aguda $(2,3 \%$ en cada grupo, $\mathrm{p}=0,99)(8)$.

\section{Recomendación del resumen ejecutivo de la ATS/ERS/JRS/ALAT julio 2015 no sugiere utilizar la combinación de la terapia $\mathrm{N}$-acetilcisteína, azatioprina y prednisona en pacientes con fibrosis pulmonar idiopática (Recomendación fuerte) (9)}

La pirfenidona es un medicamento antifibrótico oral con múltiples efectos, sin mecanismo de acción claro, con disminución en la producción de citoquinas, quimoquinas y factores de crecimiento como el TGF-B, factor de crecimiento de fibroblasto, TNF alfa, IL-1B, IL-6, CXCL12 y CCL2, como antioxidante, como inhibidor de los fibroblastos y proliferación en miofibroblastos y síntesis del colágeno. Este medicamento fue ampliamente investigado por los japoneses, con estudio en fase II que tuvo que ser parado por disminución de exacerbaciones y mejoría en la CVF con respecto al placebo (9).

El estudio de Taniguchi tuvo errores metodológicos con cambio en el objetivo principal por CVF, con disminución en la tasa de caída de la CVF (-90 $\mathrm{ml}$ vs $-160 \mathrm{ml}, \mathrm{p}=0,04)$ y mejoría en la progresión de la enfermedad $(p=0,03)$ (10) por lo cual se realizaron 2 estudios similares, multicéntricos, doble ciego, aleatorizados, controlados, con grupos poblacionales comparables; el CAPACITY 004 y 006, con 779 pacientes de 40 a 80 años, en un periodo de 72 semanas en total con objetivo primario cambio en la CVF. Los resultados fueron variables con respecto a los 2 estudios, la CVF mostró una reducción de la disminución durante las 72 semanas en 
el estudio 004 con una $\mathrm{p}=0,001$, mientras que en el 006 no mostró esa disminución. La progresión de la enfermedad con la dosis de $2.403 \mathrm{mg}$ /día en el 004 mostró una reducción del $36 \%$, pero en el 006 no y hubo mejoría en la caminata de 6 minutos en el 006 de 56 metros con respecto al placebo mientras que en el 004 no hubo disminución, con eventos adversos gastrointestinales especialmente diarrea y fotosensibilidad (11).

En el estudio fase 3 (ASCEND) con 555 pacientes con fibrosis pulmonar idiopática con pirfenidona $2.403 \mathrm{mg} /$ día o placebo por 52 semanas el objetivo primario fue el cambio en la CVF o la muerte, con reducción del $47.9 \%$ en la proporción de pacientes que tuvieron una disminución de $10 \%$ o más del predicho de la CVF o murieron y un incremento relativo del $132,5 \%$ en la proporción de pacientes que no tuvieron disminución en la CVF $(\mathrm{P}<0,001)$. El promedio de disminución en la CVF fue de $235 \mathrm{ml}$ en el grupo de la pirfenidona y $428 \mathrm{ml}$ en el grupo de placebo, con una diferencia absoluta de $193 \mathrm{ml}$ $(\mathrm{p}<0,001)$ y linear de $116 \mathrm{ml}$. La pirfenidona mejoró 50 metros la distancia recorrida con respecto al placebo $(\mathrm{P}=0,04)$ y mejoró la sobrevida y su progresión $(\mathrm{P}<0,001)(12)$.

El estudio ASCEND fue más estricto metodológicamente y escogió pacientes más severos, con fibrosis moderada en su clase funcional por su CVF y difusión. No sabemos qué pasa en pacientes más severos con duración de los síntomas de más de 52 semanas, calidad de vida y pruebas funcionales y si la debemos utilizar. Es obligatorio ver la adherencia, la seguridad del medicamento y sus eventos adversos, de ahí la necesidad de la identificación más temprana de la enfermedad hasta ahora no disponible. Es probable que los biomarcadores nos ayuden a iniciar un tratamiento, realizar un seguimiento y ver el pronóstico de la misma. No existen datos sobre la mejoría sintomática y de calidad de vida en los estudios, muy importante para el paciente y no solo la mejoría absoluta de la CVF de $116 \mathrm{ml}$ que nos muestra el estudio ASCEND, y no hay resultados sobre la disminución de la mortalidad. Es necesario hacer un control estricto de la función hepática, de las nauseas, vómito, diarrea y de la fotosensibilidad en los pacientes con el medicamento. Según la FDA la dosificación debe ser gradual y semanal (1 tableta cada 8 horas la primera semana, 2 tabletas cada 8 horas la siguiente semana y 3 tabletas cada 8 horas en adelante) para ver la tolerancia y adherencia al medicamento. Las 9 tabletas al día $(2.403 \mathrm{mg} /$ día $)$, que fue la dosis aprobada por los estudios, es difícil de lograr dada su pobre tolerancia y la costoefectividad hasta ahora no evaluada.

\section{Recomendación del resumen ejecutivo de Julio 2015 de la ATS/ERS/JRS/ALAT sugieren el uso de la pirfenidona en pacientes con fibrosis pulmonar idiopática (Recomendación moderada) (9)}

El nintedanib es un inhibidor de las tirosin kinasas. Se han visto que al inhibir estos factores de crecimiento se puede retrasar la progresión de la fibrosis pulmonar idiopática. El factor de crecimiento de los fibroblastos (FGF) y el factor derivado de las plaquetas (PDGF) están involucrados en la proliferación de fibroblastos y la deposición en la matriz extracelular, además de la diferenciación de los fibroblastos en miofibroblastos. No es claro el papel de la neovascularización y la señal del factor de crecimiento vascular endotelial (VEGF) en la fibrosis pulmonar idiopática, donde también actúa el nintedanib.

Se realizaron 2 estudios controlados doble ciego fase 2: los estudios TOMORROW, con 432 pacientes para evaluar el cambio anual en la CVF y la diminución de las exacerbaciones. Se evaluaron 4 dosis frente a placebo, permitiendo a un subgrupo de pacientes que venían recibiendo tratamiento previo seguir tomándolo como esteroides, azatioprina, $\mathrm{N}$-acetilcisteína y otros. Se encontró que la proporción de pacientes con más del $10 \%$ de disminución de la CVF anual fue menor con la dosis alta de nintedanib, 0,06 L comparado con $0,19 \mathrm{~L}$ del brazo de placebo $(p=0,004)$. Las exacerbaciones disminuyeron con cualquier dosis comparado con los controles (HR, 0,16 95\%CI, 0,04-0,70). No hubo diferencia significativa con respecto a la mortalidad entre los grupos. Hubo mayores eventos adversos especialmente diarrea y náusea en la dosis más alta de nintedanib (13). 
Surgen 2 nuevos estudios denominados INPULSIS 1 y 2 de 52 semanas de duración aletaorizados, doble ciego, controlados, con 1.066 pacientes, con nintedanib $150 \mathrm{mg}$ cada 12 horas frente a placebo en pacientes con fibrosis pulmonar idiopática. Como objetivo principal se evaluó la disminución anual del a CVF. Los resultados de la disminución de la CVF anual fue en el INPULSIS 1 fue de $125,3 \mathrm{ml}$ $(\mathrm{p}=0,001)$ y del INPULSIS 2 de $93,7 \mathrm{ml}(\mathrm{P}=0,01)$. En el INPULSIS 1 no hubo diferencia con la aparición de la exacerbación (HR: 1,15, 95\% IC, p=0,67) y en el INPULSIS 2 si hubo diferencia del nintedanib frente al placebo (HR: 0,38, 95\% IC p=0,005). En los cuestionarios de calidad de vida no hubo diferencia entre los grupos. Con respecto a los eventos adversos el más frecuente al igual que en los estudios anteriores es la diarrea, aunque menos del 5\% de los pacientes descontinuaron el medicamento (14).

\section{Recomendación del resumen ejecutivo de la ATS/ERS/JRS/ALAT Julio 2015 sugieren el uso del nintedanib en pacientes con fibrosis pulmonar idiopática (Recomendación mode- rada) (9)}

Con los resultados del nintedanib en los 3 estudios se confirma el beneficio del uso de este medicamento en la fibrosis pulmonar idiopática. Al igual que con el otro medicamento, hubo mayor rigor metodológico especialmente en los estudios de Richeldi y colaboradores quienes realizaron en el INPULSIS 1 y 2 un análisis de sensibilidad mayor especialmente con la CVF y la exacerbación para los resultados, aunque no se escogieron pacientes tan severos, con CVF mayor del $80 \%$. Llama la atención que en los subgrupos de los estudios permitieron dejar a los pacientes que venían utilizando tratamientos previos como prednisolona $15 \mathrm{mg} /$ día como criterio de inclusión. Debemos evaluar su uso en pacientes con enfermedad avanzada (CVF menor de 50\%), con tratamiento combinado. La adherencia al manejo a largo plazo hasta ahora no se ha evaluado. No hubo diferencia significativa en los cuestionarios de St George. Quizá lo más importante para el paciente fue la disminución de $125,3 \mathrm{ml}$ de la CVF y hubo algunos pacientes incluidos con diagnostico por TACAR con diagnostico de probable fibrosis pulmonar idiopática y no definitivo.

La FDA en su aprobación muestra los eventos adversos que a pesar de que se puede disminuir la dosis y ajustarla cuando se presenten, especialmente la diarrea, no hubo descontinuación en más del 5\% de los pacientes. Se debe realizar un control estricto de la función hepática e interacción medicamentosa con medicamentos que actúen en citocromo p450, vigilar el riesgo de sangrado y pacientes con enfermedad cardiovascular antes de su uso.

El trasplante pulmonar debe ser una alternativa en el manejo de estos pacientes en los cuales no debemos esperar hasta un deterioro funcional para remitirlos a un centro de trasplante. Debemos indicarlo de entrada en pacientes con CVF menor de $50 \%$, y difusión menor de $35 \%$ o en pacientes que disminuyan su CVF en más del 10\%, o su difusión de más del $15 \%$, que hayan recibido algunos de los tratamientos o la combinación de ellos y no se estabilicen o se deterioren sus síntomas. También en los que tengan más de 2 terceras partes de panalización en la TACAR. El trasplante es el único tratamiento que mejora al paciente en la parte funcional e incrementa la supervivencia de $1,5(45 \%)$ y 10 años (22\%), comparado con los resultados de los medicamentos (15).

Finalmente debemos crear centros de referencia en nuestro país para el manejo de este tipo de pacientes, para el diagnóstico precoz y manejo, igual al cáncer, con 2 o 3 medicamentos a la vez (16). Es importante identificar los diferentes fenotipos de la enfermedad para clasificar con biomarcadores su diagnóstico y pronóstico. No olvidar la parte genómica de los pacientes respondedores de los no respondedores a los tratamientos, con las alteraciones hereditarias familiares como se ven en las formas de la mutación de los telómeros, el surfactante $\mathrm{C}$ y las variantes MUC5b que van a influir en la respuesta al tratamiento. Existen en el momento en estudio nuevas moléculas contra IL13, ácido lisofosfatídico, inhibidores del factor de crecimiento del tejido conectivo (CTGF) e inhibidores de la lisil oxidasa que quizás nos ayuden al tratamiento de esta enfermedad (4). 


\section{Bibliografía}

1. Antoniou K, Margaritopoulos G, Siafakas N. Pharmacologic treatment of idiopathic pulmonary fibrosis: from the past to the future. Eur Respir Rev. 2013;22:218-91.

2. Buendia I, Caro M, Curbelo P, et al. Recomendaciones para el diagnóstico y tratamiento de la fibrosis pulmonar idiopática. Guía ALAT 2014.

3. Maher T. Idiopathic pulmonary fibrosis: pathobiology of novel approaches to treatment. Clin Chest Med. 2012;33:69-83.

4. Ahluwalia N, Shea B, Tager. New therapeutic targets in idiopathic pulmonary fibrosis. Am J Respir Crit Care Med. 2014;190(8):867-78.

5. Stuebner M, Christine D, Garcia K. Genetic interstitial lung disease. Clin Chest Med. 2012;33:95-110.

6. Demedts M, Behr J, Buhl R, et al. High dose acetylcistein in idiopathic pulmonary fibrosis. N Engl J Med. 2005;353;2229-42.

7. The idiopathic pulmonary fibrosis clinical research network. Prednisone, azatioprine, and n-acetylcistein for pulmonary fibrosis. N Engl J Med. 2012;366;1968-77.

8. Raghu G, Anstron J, King T, et al. Randomized trial of acetylcisteine in idiopathic pulmonary fibrosis. N Engl J Med. 2014.

9. Raghu G, Rochwerg B, Zhang Y. et al. An official ATS/ERS/JRS/ALAT Clinical Practice guideline: treatment of idiopathic pulmonary fibrosis: Executive Summary. Am J Respir Crit Care Med. 2015; 192:238-48.
10-Taniguchi H, Ebina M, Kondoh Y, et al. Pirfenidone in idiopathic pulmonary fibrosis. Eur Respir J. 2010;35:821-9.

11. Noble PW, Albera C, Bradford WZ, et al. Pirfenidone in patients with idiopathic pulmonary fibrosis (CAPACITY): two randomized trials. Lancet. 2011:377:1760-9.

12. King TE Jr, Bradford WZ, Castro-Bernardini S, Fagan EA, Glaspole I, Glassberg MK, et al.; ASCEND Study Group. A phase 3 trial of pirfenidone in patients with idiopathic pulmonary fibrosis. N Engl J Med. 2014;370:2083-92.

13. Richeldi L, Costabel U, Selman M, et al. Efficacy of a tyrosine kinasa inhibitor in idiopathic pulmonary fibrosis. N Engl J Med. 2011;365:1079-87.

14. Richeldi L, du Bois RM, Raghu G, Azuma A, Brown KK, Costabel U, et al.; INPULSIS Trial Investigators. Efficacy and safety of nintedanib in idiopathic pulmonary fibrosis. N Engl J Med. 2014;370:2071-82.

15. Xaubet A, Ancochea J, Bollo E et al. Normativa sobre el diagnóstico y tratamiento de la fibrosis pulmonar idiopática. Arch Bronconeumol. 2013;49(8):343-53.

16. Vancheri C, Failla M, Crimi N, Raghu G. Idiopathic pulmonary fibrosis; a disease with similarities and links to cancer biology. Eur Respir J. 2010;35:496-504

17. Raghu G, Collard H, Amstrom K. Idiopathic pulmonary fibrosis. Clinically meaningful primary endpoints in phase 3 clinical trials. Am JRespir Crit Care Med. 2012;185(10): 1044-8. 